\title{
Off Rowland spectrometer with shifted slit
}

\author{
P.S. Antsiferov ${ }^{1,2, *}$, L.A. Dorokhin ${ }^{1}$, P.V. Krainov ${ }^{1,2}$ \\ ${ }^{1}$ Institute for Spectroscopy RAS, 108840 Troitsk, Moscow, Russia \\ ${ }^{2}$ Technological Institute for Superhard and Novel Carbon Materials, 108840, Troitsk, Moscow, \\ Russia
}

\begin{abstract}
The article presents the scheme of grazing incidence spectrometer with the entrance slit shifted from the Rowland circle. This scheme allows to get the focusing surface normal to the line of site and to use the micro channel plate detector for the registration of the spectra. The results of the experimental tests are also given.
\end{abstract}

The detection of vacuum ultra violet (VUV) and soft X-ray radiation by means of micro channel plates (MCP) combines high sensitivity (quantum efficiency up to $15 \%$ ) and time resolution down to $1 \mathrm{~ns}$, which is important for the plasma diagnostics applications. The usage of MCP detectors is optimal in the case of normal incidence of radiation. As it was shown still in [1], the shift of the entrance slit from the Rowland circle allows to change the angle of the radiation incidence on the surface of spectral focusing, see Fig.1.

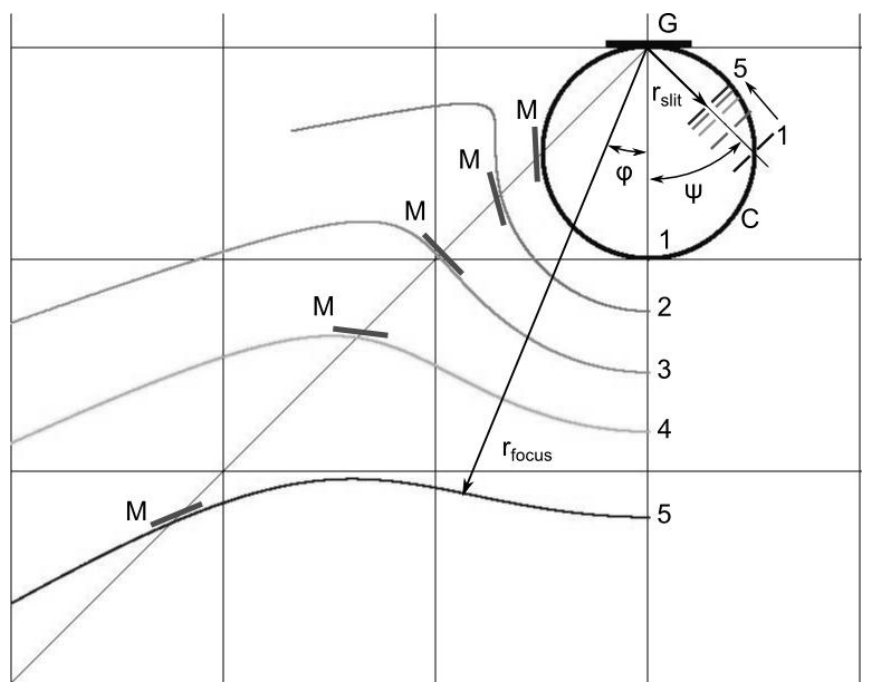

Fig. 1. $\mathrm{G}$ - diffraction grating, $\mathrm{C}-$ Rowland circle. The entrance slit positions $1-5$ correspond to the focusing curves $1-5$ according [1]. $\mathrm{M}$ - correspondent positions of the entrance surface of MCP.

${ }^{*}$ Corresponding author: Ants@isan.troitsk.ru 
The particular scheme of the spectrometer for the wavelength range 100-300 Angstroms with MCP detector is given in Fig.2. The estimated spectral resolution $\lambda / \delta \lambda$ is in the range 300-700 [2]. The main parameters of the spectrometer: diffraction grating grooves density $600 \mathrm{gr} / \mathrm{mm}$, diffraction grating radius of curvature $1 \mathrm{~m}$, the entrance slit width $10 \mu \mathrm{m}$, the grazing angle $4^{0}$, the distance slit - grating $3.3 \mathrm{~cm}$, the working size of the grating $6 \mathrm{~mm}$, the distance MCP - grating $33.1 \mathrm{~cm}$.

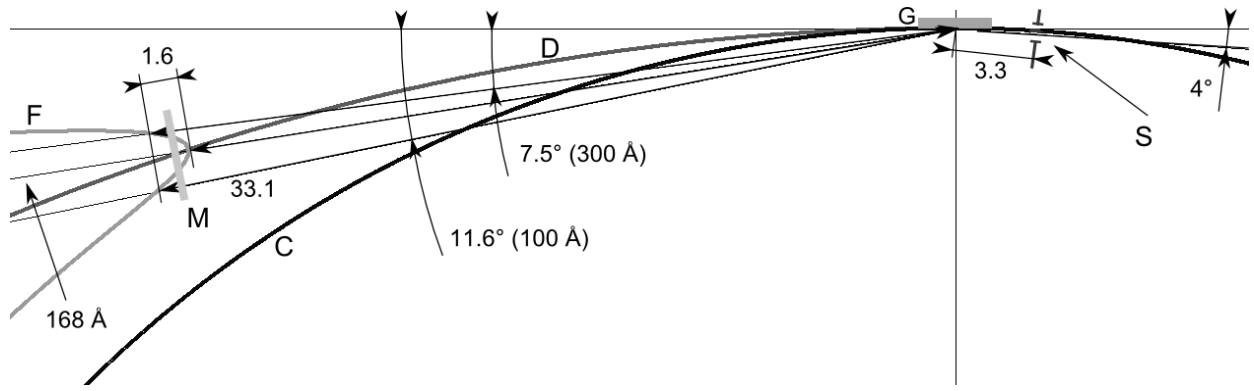

Fig. 2. The drawing of the proposed off-Rowland spectrometer with shifted slit. G - diffraction grating, R- Rowland circle, D - double Rowland circle, $\mathrm{S}$ - entrance slit, $\mathrm{F}$ - focusing curve, $\mathrm{M}$ - the entrance surface of the MCP.

An example of the VUV spectrum of multiply charged ions of Ar and the spectral resolution data are given in Fig.3. The deterioration of the experimental spectral resolution in comparison with calculated [2] can be explained by the influence of the apparatus function of MCP detector.
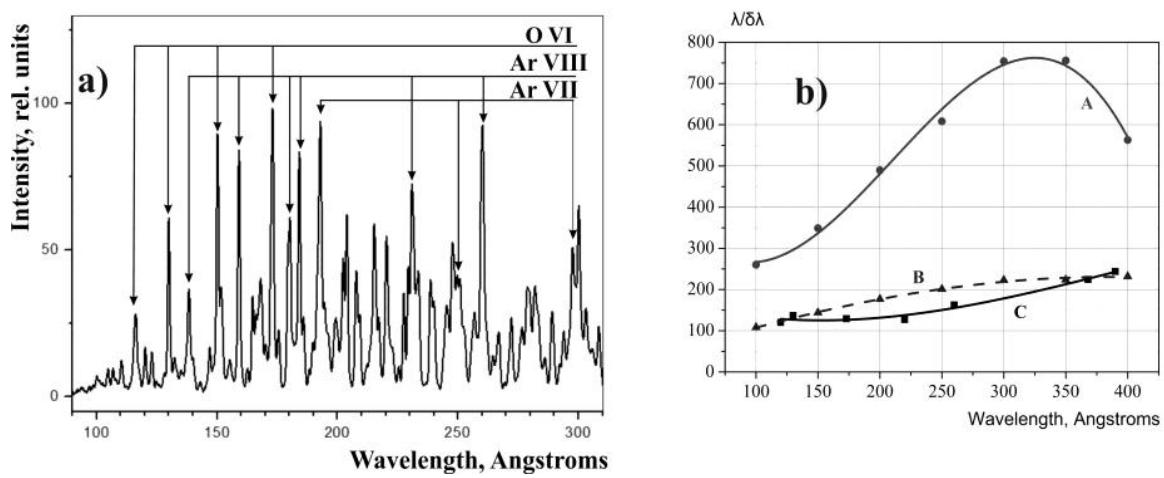

Fig. 3. a) the example of the spectrum, obtained with the help of off- Rowland spectrometer with shifted slit, b) A - the results of the numerical estimation [2] of the spectral resolution, B - the degradation of the spectral resolution by the influence of $100 \mu \mathrm{m}$ apparatus function, $\mathrm{C}-$ the experimental results.

The work was supported by RFBR grant No 15-02-04411a.

\section{References}

1. W. Baily, Philisophical Magazine. Series 5 15, 183 (1883)

2. P.S. Antsiferov, L.A. Dorokhin, P.V. Krainov, Review of Scientific Instruments 87, 053106 (2016) 